\title{
A Practical Control Strategy for the Maglev Self-Excited Resonance Suppression
}

\author{
Jinhui Li, ${ }^{1,2}$ Dong Fang, ${ }^{1}$ Ding Zhang, ${ }^{1}$ Ying Cai, ${ }^{1} \mathrm{Qi} \mathrm{Ni},{ }^{1}$ and Jie $\mathrm{Li}^{2}$ \\ ${ }^{1}$ Science and Technology on Near-Surface Detection Laboratory, Wuxi 214035, China \\ ${ }^{2}$ College of Mechatronics Engineering and Automation, National University of Defense Technology, Changsha 410073, China \\ Correspondence should be addressed to Jinhui Li; li_jinhui@126.com
}

Received 17 March 2016; Accepted 5 June 2016

Academic Editor: Kishin Sadarangani

Copyright (c) 2016 Jinhui Li et al. This is an open access article distributed under the Creative Commons Attribution License, which permits unrestricted use, distribution, and reproduction in any medium, provided the original work is properly cited.

\begin{abstract}
This paper addresses the control strategy for the suppression of maglev vehicle-bridge interaction resonance, which worsens the ride comfort of vehicle and degrades the safety of the bridge. Firstly, a minimum model containing a flexible bridge and ten levitation units is presented. Based on the minimum model, we pointed out that magnetic flux feedback instead of the traditional current feedback is capable of simplifying the block diagram of the interaction system. Furthermore, considering the uncertainty of the bridge's modal frequency, the stability of the interaction system is explored according to an improved root-locus technique. Motivated by the positive effects of the mechanical damping of bridges and the feedback channels' difference between the levitation subsystem and the bridge subsystem, the increment of electrical damping by the additional feedback of vertical velocity of bridge is proposed and several related implementation issues are addressed. Finally, the numerical and experimental results illustrating the stability improvement are provided.
\end{abstract}

\section{Introduction}

Compared with the conventional railway systems, the electromagnetic maglev system has advantages of lower noise, less exhaust fumes emission, less maintenance cost, and the ability to climb steeper slopes, which is a new kind of urban transport that has been widely concerned in recent years [1-3].

However, when the maglev is suspended upon the bridge, standing still or moving at very slow speed, the bridge and vehicle may vibrate continuously with oversized amplitude, which is called maglev self-excited vibration. The self-excited vibration degrades the safety of the bridge and worsens the ride comfort of vehicle, which is a burning issue to be solved [4-6].

Up to the present, extensive investigations on the principle underlying the maglev self-excited vibration have been reported. Alberts et al. $[7,8]$ pointed out that the American Maglev Technologies system achieved successful levitation in Florida on a bridge mounted to the earth on a concrete foundation but later encountered difficulties in achieving stable levitation when the vehicle was moved to the bridge installed on the Old Dominion University campus. They believed that the over flexibility of the bridge on the Old Dominion University campus was the main reason. Wang et al. [9] pointed out that the self-excited vibration is due to the improper frequency relationship between various components of the system. Zhang et al. [10,11] studied the signal delay's influences on the stability of nonlinear levitation system. The analysis shows that the system will undergo a periodic vibration when the time-delay reaches a critical value.

Recently, more works are focused on the engineering solutions of self-excited vibration. Generally, the solutions tend to be divided into two groups. The first group is to optimize the parameters of the bridge system, including enlarging the mass per meter [12], enlarging the modal damping [13,14], and decreasing the modal frequencies $[15,16]$.

To some extent, these optimizations are capable of avoiding the self-excited vibration. In engineering, the mass increment of bridge was widely adopted. However, it raises the initial cost significantly. The modal damping ratio is mainly determined by the bridge's materials. Hence, the increment of modal damping ratio is a theoretical method and unavailable 


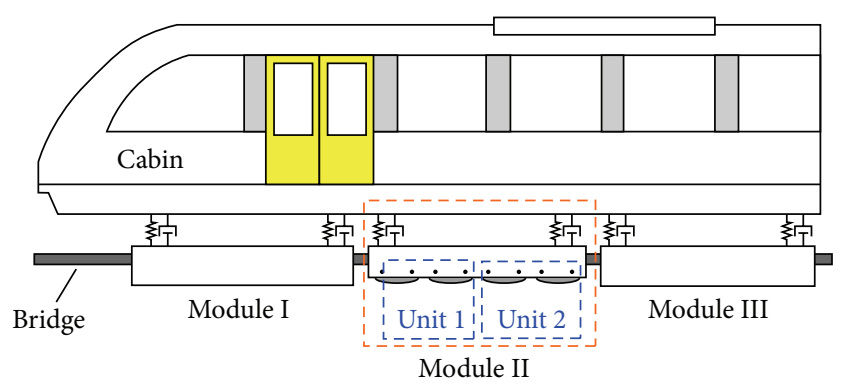

FIgURE 1: The side views of CMS04 maglev system.

in engineering. Decreasing the modal frequencies of bridges is unsuitable for the completed maglev routes.

The other group is to improve the control strategy of levitation system, including optimization of the parameters and minimization of the time-delay of feedback channels [17], virtual tuned mass damper algorithm [6], and the virtual energy harvester algorithm [18]. They believed that these control strategies are capable of avoiding the self-excited vibration for the given bridge, and the robustness to bridges with different modal frequencies awaits further research.

To analyze the robustness of the control scheme to bridges with different modal frequencies, in this paper, the magnetic flux feedback instead of the traditional current feedback is proposed to simplify the block diagram of the vehicle-bridge interaction system, and an improved root-locus technique corresponding to the modal frequencies is explored.

Furthermore, to avoid the instability of the bridge with improper modal frequency and minor modal damping, a practical control scheme is explored from the perspective of theoretical source and engineering implementation. Finally, its validity is checked experimentally by the real and full-sized maglev system.

The research reported here is engineering-oriented. The purpose is to develop a practical control strategy that is capable of eliminating the self-excited vibration and is applicable to a real maglev system.

\section{Modeling of Vehicle-Bridge Interaction System}

Considering the complexity of the self-excited vibration, an overall dynamic model of the interaction system with details, which is shown in Figure 1, may result in a difficult analysis to draw useful conclusions. Here, a minimum interaction model containing the quintessential parts, a flexible bridge, and some levitation units is presented.

2.1. Modeling of Bridge. In this section, the maglev bridge is simplified as a Bernoulli-Euler beam due to the fact that the length of the bridge is much larger than the size of other dimensions. The nonlinearity behaviors of the bridge are neglected because the amplitude of the vibration is sufficiently small when compared with the span of bridge [18].

For the maglev vehicle, the dynamics of sprung mass and the coupling force between different electromagnets are

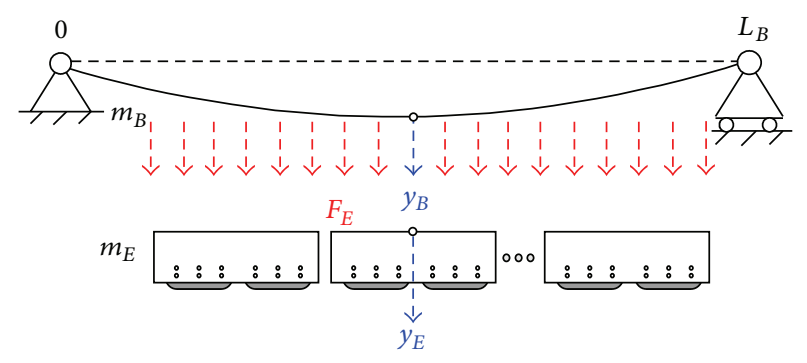

FIGURE 2: The minimum model of maglev vehicle-bridge system.

neglected due to the isolation effect of air-spring and antiroll beams. Based on the above assumptions, the minimum interaction model is shown in Figure 2. Variable $L_{B}$ is the span of the bridge.

Furthermore, the motion of bridge is described by the following differential equation [6]:

$$
E I_{B} \frac{\partial^{4} y_{B}(x, t)}{\partial x^{4}}+\rho_{B} \frac{\partial^{2} y_{B}(x, t)}{\partial t^{2}}=F_{E}(x, t),
$$

where $x$ is the axial coordinate of the bridge, $y_{B}$ is the vertical displacement of bridge, $t$ is the time, $E I_{B}$ is the bending rigidity, $\rho_{B}$ is the mass per meter of bridge, and $F_{E}(x, t)$ is the electromagnetic force acting on the bridge.

For the simply supported concrete beam, $k$ th modal frequency $\omega_{B k}$ and modal shape functions $\phi_{B k}(x)$ are $[19,20]$

$$
\begin{aligned}
\omega_{B k} & =\lambda_{B k}^{2} \sqrt{\frac{E I_{B}}{\rho_{B}}}, \\
\phi_{B k}(x) & =\sin \lambda_{B k} x,
\end{aligned}
$$

where $\lambda_{B k}=k \pi / L_{B}$. Using the modal superposition method, the solutions of (1) may be expressed as

$$
y_{B}(x, t)=\sum_{k=1}^{\infty} \phi_{B k}(x) q_{B k}(t) .
$$

Here, $q_{B k}(t)$ is the time-varying amplitude of $k$ th modal displacement. When considering the mechanical damping of the bridge, substituting (3) into (1), multiplying both sides of the aforementioned resultant equation by $\phi_{B k}(x)$, and then integrating both sides from 0 to $L_{B}$, it gives

$$
\begin{array}{r}
\ddot{q}_{B k}(t)+2 \xi_{B k} \omega_{B k} \dot{q}_{B k}(t)+\omega_{B k}^{2} q_{B k}(t) \\
=2 m_{B}^{-1} \cdot \int_{0}^{L_{B}} \phi_{B k}(x) F_{E}(x, t) d x .
\end{array}
$$

According to the observation of the low-speed maglev test base of china, the maglev self-excited vibration is mainly evoked by the first modal of bridge. Hence, the stability of the first modal of bridge should be emphasized and the higher modals may be neglected temporarily. In this case, (4) is simplified as

$$
\begin{aligned}
& \ddot{q}_{B}(t)+2 \xi_{B} \omega_{B} \dot{q}_{B}(t)+\omega_{B}^{2} q_{B}(t) \\
& =2 m_{B}^{-1} \cdot \int_{0}^{L_{B}} \phi_{B}(x) F_{E}(x, t) d x .
\end{aligned}
$$


Here, $q_{B}(t)$ is the first modal displacement, $\phi_{B}(x)$ is the first modal shape functions, and $\omega_{B}$ is the first modal frequency of bridge. Multiplying both sides of the resultant equation by $\phi_{B}(x)$, it gives

$$
\begin{aligned}
\ddot{y}_{B} & (x, t)+2 \xi_{B} \omega_{B} \dot{y}_{B}(x, t)+\omega_{B}^{2} y_{B}(x, t) \\
& =2 \phi_{B}(x) m_{B}^{-1} \cdot \int_{0}^{L_{B}} \phi_{B}(x) F_{E}(x, t) d x .
\end{aligned}
$$

Assuming that the number of levitation units suspended on the bridge is $n$, the length of levitation unit is $L_{E}$, and the left axial location of $i$ th levitation unit is $x_{L E i}$, in this case, (6) may be rewritten as

$$
\begin{aligned}
\ddot{y}_{B} & (x, t)+2 \xi_{B} \omega_{B} \dot{y}_{B}(x, t)+\omega_{B}^{2} y_{B}(x, t) \\
& =2 \phi_{B}(x) m_{B}^{-1} \cdot \sum_{i=1}^{n} \int_{x_{L E i}}^{x_{L E i}+L_{E}} \phi_{B}(x) F_{E}(x, t) d x .
\end{aligned}
$$

In this section, the nonlinearity behavior of the bridge is neglected because the amplitude of the vibration is sufficiently small when compared to the span of bridge. According to [17], the fundamental component of electromagnetic force $F_{E}(x, t)$ is proportional to its shape function $\phi_{B}(x)$. In this case, it gives

$$
\begin{gathered}
\ddot{y}_{B}(x, t)+2 \xi_{B} \omega_{B} \dot{y}_{B}(x, t)+\omega_{B}^{2} y_{B}(x, t)=2 \phi_{B}(x) \\
\cdot m_{B}^{-1} \phi^{-1}\left(0.5 L_{B}\right) \\
\cdot \sum_{i=1}^{n} \int_{x_{L E i}}^{x_{L E i}+L_{E}} \phi_{B}^{2}(x) d x \cdot F_{E}\left(0.5 L_{B}, t\right) .
\end{gathered}
$$

With regard to special case $x=0.5 L_{B}$, it gives that

$$
\ddot{y}_{B}(t)+2 \xi_{B} \omega_{B} \dot{y}_{B}(t)+\omega_{B}^{2} y_{B}(t)=\sigma \cdot \frac{F_{E}(t)}{m_{B}} .
$$

Here, $\sigma=2 \phi_{B}(x) \phi^{-1}\left(0.5 L_{B}\right) \sum_{i=1}^{n} \int_{x_{L E i}}^{x_{L E i}+L_{E}} \phi_{B}^{2}(x) d x$, variable $y_{B}(t)$ is the modal displacement and variable $F_{E}(t)$ is the electromagnetic force of the levitation unit at the axial location of $x=0.5 L_{B}$.

2.2. Modeling of Levitation System with Current Feedback. Suppose the turns of a single electromagnet are $N$, the pole area is $A$, and the magnetic permeability of vacuum is $\mu_{0}$. For a single electromagnet, the dynamic equations related voltage $u(t)$ and electromagnetic force $F_{E}(t)$ are

$$
\begin{aligned}
u(t) & =2 R i(t)+\mu_{0} A N^{2}\left[\dot{i}(t) \delta^{-1}(t)-\frac{i(t) \dot{\delta}(t)}{\delta^{2}(t)}\right], \\
F_{E}(t) & =\frac{0.5 \mu_{0} A N^{2} i^{2}(t)}{\delta^{2}(t)},
\end{aligned}
$$

where $R$ is the resistance, $i(t)$ is the current of electromagnet, and $\delta(t)$ is the averaged electrical clearance between the bridge and related electromagnet. Considering the vibration isolation effect of air-spring, the dynamics of sprung mass is neglected. Then the movement of electromagnet is

$$
m_{E} \ddot{y}_{E}(t)=-F_{E}(t)+\left(m_{C}+m_{E}\right) g,
$$

where $y_{E}(t)$ is the vertical displacement of electromagnet, $g$ is the acceleration of gravity, $m_{C}$ is the sprung mass, and $m_{E}$ is the mass of electromagnet. According to (12), it can be seen that steady current $i_{0}$ and steady voltage $u_{0}$ of electromagnet are

$$
\begin{aligned}
& i_{0}=\delta_{0} \sqrt{\frac{2\left(m_{C}+m_{E}\right) g}{\left(\mu_{0} N^{2} A\right)}}, \\
& u_{0}=2 R i_{0} .
\end{aligned}
$$

Here, variable $\delta_{0}$ is the steady levitation gap. Due to its clear physical meaning and excellent performance in maglev engineering, the cascaded-controller, whose inner loop adopts the feedback of current negative feedback, is widely applied $[17,18]$. The control scheme is

$$
\begin{aligned}
i_{E}(t) & =\bar{k}_{p}\left[y_{E}(t)-y_{B}(t)\right]+\bar{k}_{d} \dot{y}_{E}(t)+\bar{k}_{a} \ddot{y}_{E}(t), \\
u(t) & =\bar{k}_{C}\left[i_{E}(t)-i(t)\right]+u_{0},
\end{aligned}
$$

where $i_{E}(t)$ is the desired current of the electromagnet and $\bar{k}_{p}, \bar{k}_{d}, \bar{k}_{a}, \bar{k}_{C}$ are the control parameters. It has been observed that the self-excited vibration occurs when the vehicle is suspended upon the bridge, standing still or moving at very slow speed. When the vibration amplitude of the bridge is sufficiently small, the maglev vehicle-bridge interaction system is quasistatic.

When examining the stability of the interaction system around the equilibrium point, the linearized model may be applied to simplify the analysis process without introducing noticeable errors. Considering the linear additive property of the linearized model, the expected current $i_{E}(t)$ may be divided into two parts, $i_{E 1}(t)$ and $i_{E 2}(t)$. Variable $i_{E 1}(t)$ is the total feedback of the electromagnet's states and $i_{E 2}(t)$ is the total feedback of the bridge's states. In this case, (14) may be rewritten as

$$
\begin{aligned}
& i_{E 1}(t)=\bar{k}_{p} y_{E}(t)+\bar{k}_{d} \dot{y}_{E}(t)+\bar{k}_{a} \ddot{y}_{E}(t), \\
& i_{E 2}(t)=-\bar{k}_{p} y_{B}(t) .
\end{aligned}
$$

Correspondingly, control voltage $u(t)$ is divided into two parts, $u_{1}(t)$ and $u_{2}(t)$ :

$$
\begin{aligned}
& u_{1}(t)=\bar{k}_{C}\left[i_{E 1}(t)-i_{1}(t)\right], \\
& u_{2}(t)=\bar{k}_{C}\left[i_{E 2}(t)-i_{2}(t)\right] .
\end{aligned}
$$

In this case, the block diagram of the maglev vehiclebridge interaction system is shown in Figure 3.

Block "EM" represents the balance equation (10) of control voltage and current, $F_{i}=0.5 \mu_{0} A N^{2} i_{0} \delta_{0}^{-2}, F_{\delta}=$ $0.5 \mu_{0} A N^{2} i_{0}^{2} \delta_{0}^{-3}$, which are the partial derivative of levitation force $F_{E}(t)$ to current $i(t)$ and clearance $\delta(t)$ around the equilibrium point, correspondingly. 


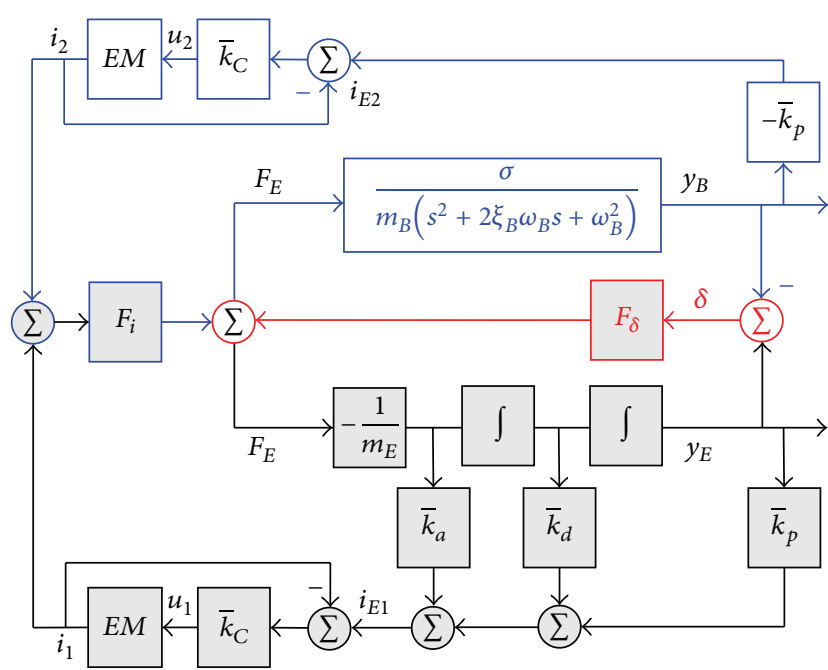

FIGURE 3: The block diagram with current feedback.

According to (11), the electromagnetic force is determined jointly by the current and levitation gap. In this case, the electromagnetic force, the current, and levitation gap interacted with each other, which results in the block diagram that is more complex and deranged.

2.3. Modeling of Levitation System with Flux Feedback. As we all know, except for (11), the electromagnetic force may be rewritten as

$$
F_{E}(t)=2 A \mu_{0}^{-1} \cdot B^{2}(t) .
$$

Correspondingly, the voltage balance equation is

$$
u(t)=2 N A \dot{B}(t)+\frac{4 R \delta(t) B(t)}{\left(\mu_{0} N\right)} .
$$

According to (18) and (19), the electromagnetic force is determined solely by the magnetic flux and the voltage balance equation is briefer than (10), which may be conductive for the active control of levitation system. To prove it, the cascadedcontroller is adopted as well:

$$
\begin{aligned}
B_{E}(t) & =k_{p}\left[y_{E}(t)-y_{B}(t)\right]+k_{d} \dot{y}_{E}(t)+k_{a} \ddot{y}_{E}(t), \\
u(t) & =k_{B}\left[B_{E}(t)-B(t)\right]+u_{D C},
\end{aligned}
$$

where $B_{E}(t)$ is the desired magnetic flux of the levitation gap and $k_{p}, k_{d}, k_{a}, k_{B}$ are the control parameters. Similarly, expected magnetic flux $B_{E}(t)$ is divided into two parts, $B_{E 1}(t)$ and $B_{E 2}(t)$. In this case, (14) may be rewritten as

$$
\begin{aligned}
& B_{E 1}(t)=k_{p} y_{E}(t)+k_{d} \dot{y}_{E}(t)+k_{a} \ddot{y}_{E}(t), \\
& B_{E 2}(t)=-k_{p} y_{B}(t) .
\end{aligned}
$$

Correspondingly, control voltage $u(t)$ is divided into two parts, $u_{1}(t)$ and $u_{2}(t)$ :

$$
\begin{aligned}
& u_{1}(t)=k_{B}\left[B_{E 1}(t)-B_{1}(t)\right], \\
& u_{2}(t)=k_{B}\left[B_{E 2}(t)-B_{2}(t)\right],
\end{aligned}
$$

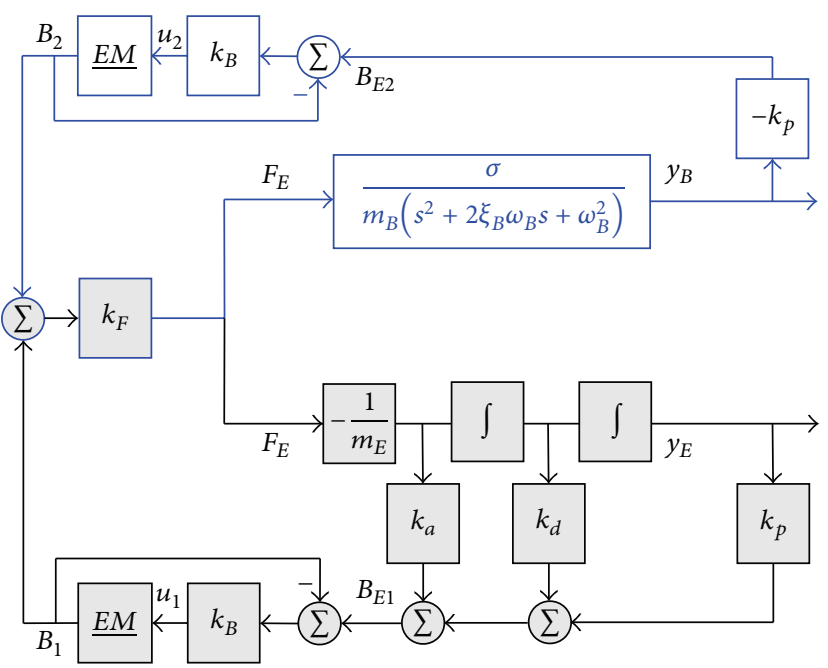

FIGURE 4: The block diagram with magnetic flux feedback.

where $k_{F}=\sqrt{8\left(m_{C}+m_{E}\right) A g / \mu_{0}}$. Comparing Figure 4 with Figure 3 , it can be seen that the magnetic flux feedback is capable of simplifying the block diagram of the maglev vehicle-bridge interaction system, which is promising and adopted to avoid the self-excited vibration.

\section{Stability Analysis of the Interaction System}

The stability of the levitation system itself is a necessary condition for the suppression of the self-excited vibration of the maglev vehicle-bridge interaction system.

3.1. Stability of the Levitation System Itself. In light of Figure 4, when the flexibility of bridge is ignored $(\sigma=0)$, the transfer function of the levitation system from electromagnetic force $F_{E}(t)$ to displacement of electromagnet $y_{E}(t)$ is

$$
T_{1}(s)=\frac{F_{E}(s)}{y_{E}(s)}=\frac{G_{E}(s)}{\left(1-G_{E}(s) H_{E}(s)\right)},
$$

where

$$
\begin{aligned}
& G_{E}(s)=\frac{-1}{m_{E} s^{2}}, \\
& H_{E}(s)=\frac{k_{B} k_{F}\left(k_{a} s^{2}+k_{p}+k_{d} s\right)}{\left(2 N A s+k_{B}\right)} .
\end{aligned}
$$

The characteristic equation of transfer function $T_{1}(s)$ is

$$
\begin{aligned}
\Delta_{1}= & 2 N A m_{E} s^{3}+\left(m_{E} k_{B}+k_{B} k_{F} k_{a}\right) s^{2}+k_{B} k_{F} k_{d} s \\
& +k_{B} k_{F} k_{p} .
\end{aligned}
$$

For the levitation subsystem, positive parameters $k_{p}, k_{d}$, $k_{a}, k_{B}$ are adjustable. According to the Routh-Hurwitz stability criterion, the levitation system itself is stable on condition that (26) is satisfied when the noise level of signals is not considered:

$$
\left(m_{E} k_{B}+k_{B} k_{F} k_{a}\right) k_{d}>2 N A m_{E} k_{p}
$$


3.2. Stability of the Interaction System. When considering the flexibility of the bridge, the transfer function of the maglev vehicle-bridge interaction system from electromagnetic force $F_{E}(t)$ to displacement of electromagnet $y_{E}(t)$ is

$$
T_{2}(s)=\frac{G_{B}(s)}{\left(1-G_{E}(s) H_{E}(s)-G_{B}(s) H_{B}(s)\right)},
$$

where

$$
\begin{aligned}
& G_{B}(s)=-\frac{\sigma m_{B}^{-1}}{\left(s^{2}+2 \xi_{B} \omega_{B} s+\omega_{B}^{2}\right)}, \\
& H_{B}(s)=k_{B} k_{F} \cdot \frac{\bar{k}_{p}}{\left(2 N A s+k_{B}\right)} .
\end{aligned}
$$

The characteristic equation of transfer function $T_{2}(s)$ is

$$
a_{5} s^{5}+a_{4} s^{4}+a_{3} s^{3}+a_{2} s^{2}+a_{1} s+a_{0}=0,
$$

where $a_{5}=2 N A m_{B} m_{E}, a_{4}=m_{B} m_{E}\left(k_{B}+4 N A \xi_{B} \omega_{B}\right)+$ $k_{B} k_{F} m_{B} k_{a}, a_{3}=m_{B} m_{E}\left(2 k_{B} \xi_{B} \omega_{B}+2 N A \omega_{B}^{2}\right)+$ $k_{B} k_{F} m_{B}\left(2 k_{a} \xi_{B} \omega_{B}+k_{d}\right), a_{2}=k_{B} \omega_{B}^{2} m_{B} m_{E}+\sigma m_{E} k_{B} k_{F} k_{p}$ $+k_{B} k_{F} m_{B}\left(k_{p}+2 k_{d} \xi_{B} \omega_{B}+k_{a} \omega_{B}^{2}\right), a_{0}=k_{B} k_{F} m_{B} k_{p} \omega_{B}^{2}$, and $a_{1}=k_{B} k_{F} m_{B}\left(k_{d} \omega_{B}^{2}+2 k_{p} \xi_{B} \omega_{B}\right)$.

Generally, the characteristic roots may be denoted as $x_{1,2}=R_{1} \pm j I_{1}, x_{3,4}=R_{2} \pm j I_{2}$, and $x_{5}=R_{3}$. If three real parts $R_{1}, R_{2}$, and $R_{3}$ all are negative for the given modal frequency of bridge, the interaction system is stable and the self-excited vibration will be avoided.

However, according to (29), the characteristic equation of the maglev vehicle-bridge interaction system is complex. When considering the adaptability to more bridges with different modal frequency, the calculation of the characteristic roots will be more difficult.

To explore the relationship between the varying modal frequency and the three real parts, the root-locus method is adopted manually by the commercial software MATLAB2012a. When the parameters are set as $k_{p}=1000, k_{d}=$ $30, k_{a}=0.4, k_{B}=30$, and $N=360$, the three real parts corresponding to varying modal frequency $\omega_{B}$ are shown in Figure 5.

When modal damping ratio $\xi_{B}$ is set as 0.005 , it can be seen that $R_{2}$ and $R_{3}$ are negative along the all axes of the modal frequency. However, real part $R_{1}$ is positive when the bridge's modal frequency belongs to interval [ 67.3118 .7$]$ rad/s. This is to say, control scheme (20) is incapable of avoiding the instability if the modal frequency falls in this interval. Hence, control scheme (20) should be improved to avoid the selfexcited vibration.

\section{Solutions to Avoid the Vibration}

4.1. Theoretical Exploration. Pang et al. and Hong and Li pointed out that enlarging the modal damping of bridge is beneficial for avoidance of the self-excited vibration $[13,14]$, which may be verified from the perspective of characteristic roots as well. When modal damping ratio $\xi_{B}$ is set as 0.02 and the other parameters are unchanged, real parts $R_{1}, R_{2}$, and $R_{3}$

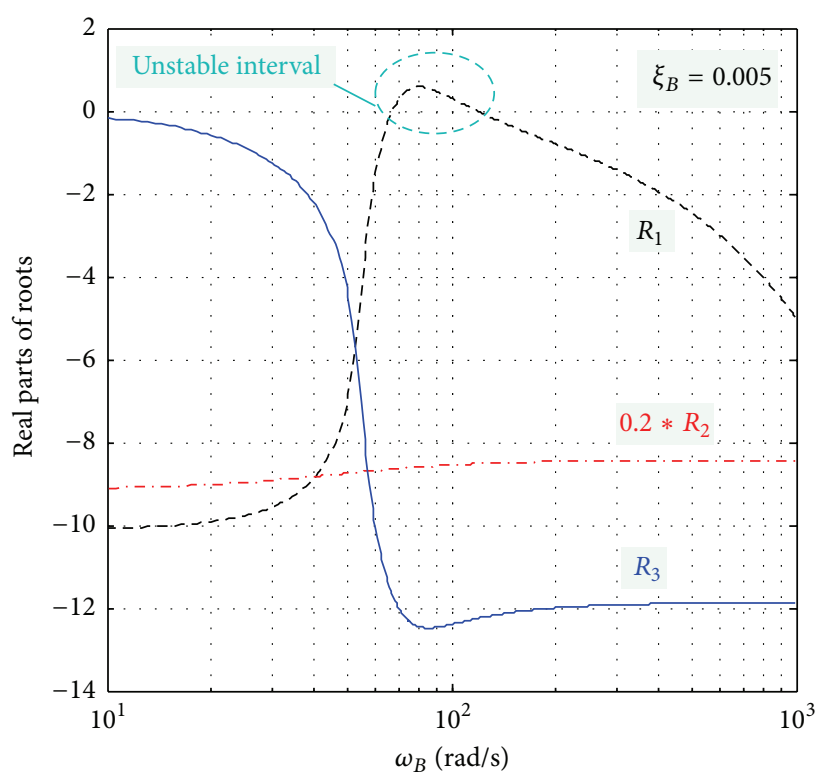

FIGURE 5: The three real parts corresponding to varying modal frequency $\omega_{B}$ when $\xi_{B}=0.005$.

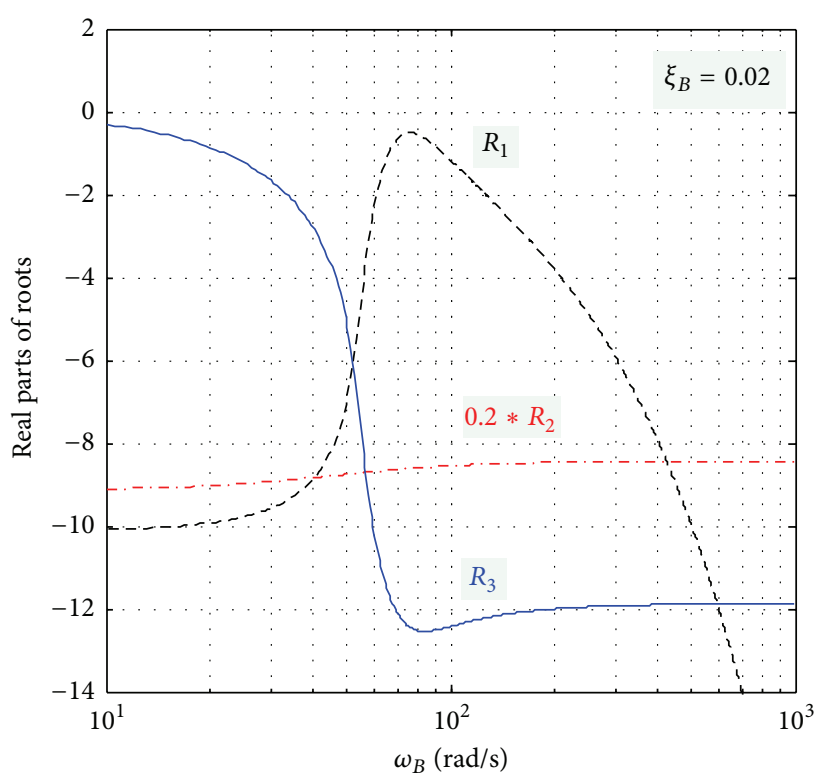

FIGURE 6: The three real parts corresponding to varying modal frequency $\omega_{B}$ when $\xi_{B}=0.02$.

are shown in Figure 6. It can be seen that three real parts, $R_{1}$, $R_{2}$, and $R_{3}$, are all negative, and the self-excited vibration is avoided.

The mechanical damping of bridges is determined by its material. To a certain extent, the bridge's stability problem could not be solved by enlarging its mechanical damping. Even so, it provides us some inspiration to avoid the selfexcited vibration by improving the damping characteristics of the bridge subsystem.

Besides, according to Figure 4, for the levitation subsystem, the displacement, velocity, and acceleration signals of 


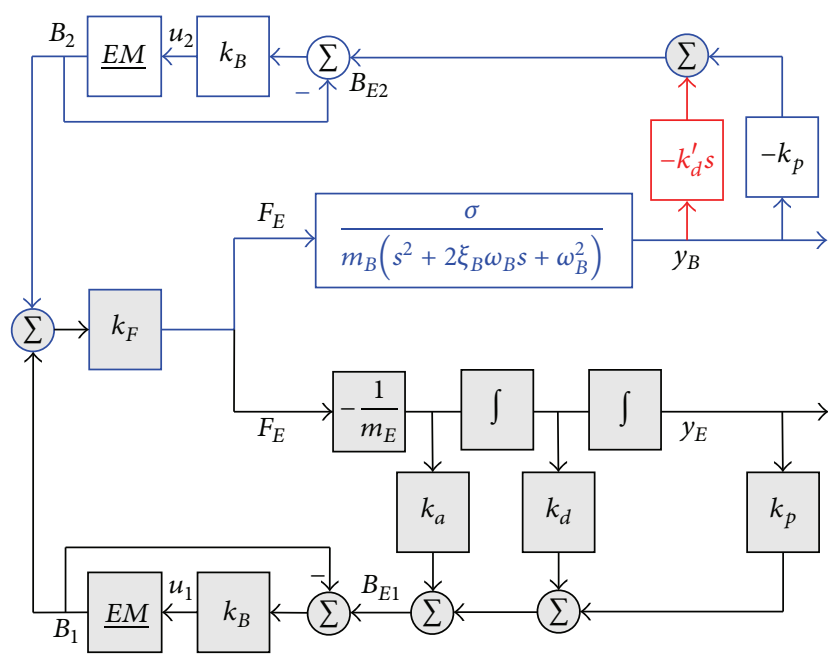

FIGURE 7: The block diagram with the bridge's velocity feedback.

electromagnet are fed back together. However, for the bridge subsystem, only the displacement information is fed back solely.

Motivated by the positive effects of the mechanical damping and the difference of the feedback channels, we guess that the feedback of the bridge's vertical velocity is available to improve the stability of the maglev vehicle-bridge interaction system. In this case, the expected magnetic flux is updated as

$$
\begin{aligned}
& B_{E 1}(t)=k_{p} y_{E}(t)+k_{d} \dot{y}_{E}(t)+k_{a} \ddot{y}_{E}(t), \\
& B_{E 2}(t)=-k_{p} y_{B}(t)-k_{d}^{\prime} \dot{y}_{B}(t) .
\end{aligned}
$$

Variable $k_{d}^{\prime}$ is the feedback gain of the bridge's velocity. Correspondingly, the block diagram with the feedback of the bridge's velocity is shown in Figure 7.

To explore the validity of control scheme (30), modal damping ratio $\xi_{B}$ is set as 0.005 and gain $k_{d}^{\prime}$ is set as 15 . In this case, real parts $R_{1}, R_{2}$, and $R_{3}$ are shown in Figure 8. It can be seen that the real parts are all negative no matter how large the modal frequency of bridge is.

Hence, we conclude that the addition of the bridge's velocity feedback to control scheme (20) is capable of avoiding the self-excited vibration theoretically.

4.2. Engineering Implementation. In light of (30), its engineering implementation that relies on the vertical velocity of the bridge, which, however, is unavailable due to the lack of sensors, so that the real-time estimation of the velocity of bridge at the location of the electromagnets is essential.

In a real maglev system, two real-time signals, including levitation gap $\delta(t)=y_{E}(t)-y_{B}(t)$, which is measured by an eddy current gap sensor, and acceleration of electromagnet $a_{E}(t)=\ddot{y}_{E}(t)$, which is detected by an accelerometer, are available.

Theoretically, the velocity signal of the electromagnet may be obtained by the integration of its acceleration signal, and

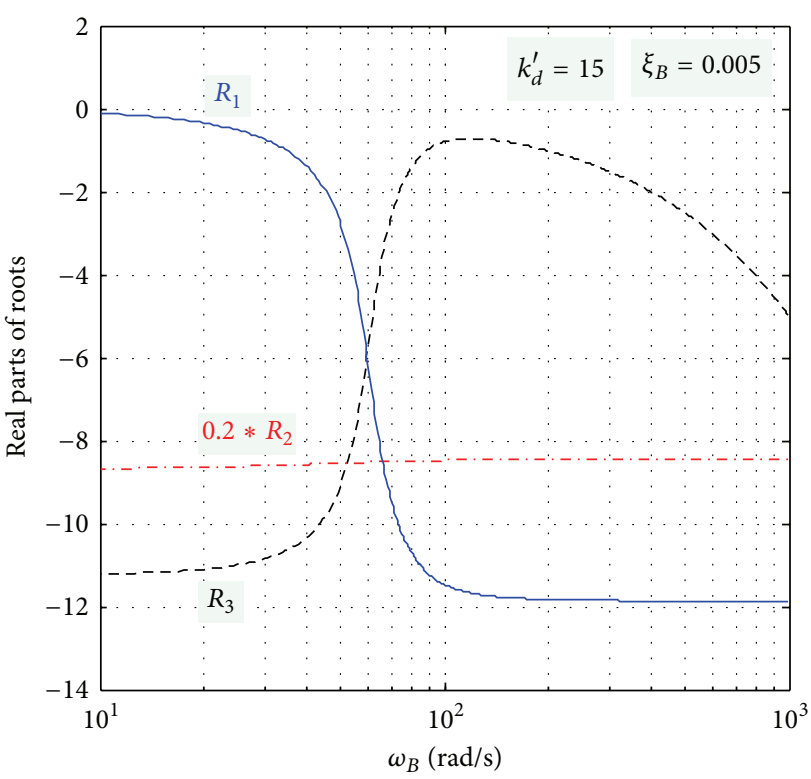

FIGURE 8: The real parts of characteristic roots when $\xi_{B}=0.005$ and $k_{d}^{\prime}=15$.

the derivation of the levitation gap may be acquired by its differentiation:

$$
\begin{aligned}
\dot{y}_{E}(t) & =\int_{\tau=0}^{t} a_{E}(\tau) d \tau, \\
\dot{\delta}(t) & =\dot{y}_{E}(t)-\dot{y}_{B}(t) .
\end{aligned}
$$

Further, the velocity signal of the bridge may be given as

$$
\dot{y}_{B}(t)=\int_{\tau=0}^{t} a_{E}(\tau) d \tau-\dot{\delta}(t) .
$$

However, in maglev engineering, owing to the leakage flux of electromagnet and the pulse of the chopper, levitation gap signal $\delta(t)$ is severely polluted. The differentiation signal of levitation gap is whelmed by the noise. To attenuate it, a low-pass filter should be added.

Besides, as for acceleration signal $a_{E}(t)$, its direct component is nonzero and uncertain due to its imperfect installation. The saturation phenomenon of integration may appear if the idea integrator is adopted. To avoid it, the self-capturing integrator is proposed. In this case, the vertical velocity of bridge may be estimated out by the following filter:

$$
\bar{v}_{B}(s)=\frac{s^{2}+2 \omega_{1} s}{s^{2}+2 \omega_{1} s+\omega_{1}^{2}} \cdot \frac{1}{s} a_{E}(s)-\frac{1}{\tau_{1} s+1} \cdot s \delta(s),
$$

where $\bar{v}_{B}(s)$ is the estimated velocity of bridge, $\omega_{1}$ is the bandwidth of self-capturing integrator, and $\tau_{1}$ is the time constant of the quasidifferentiator. Generally, bandwidth $\omega_{1}$ should be large enough to attenuate the low-frequency disturbance and uncertainty. Meanwhile, bandwidth $\omega_{1}$ should be small enough to ensure the high-frequency's correspondence between the self-capturing integrator and ideal first-order integrator. In this work, $\omega_{1}=2$ and $\tau_{1}=0.001$. 


\section{Numerical and Experimental Validation}

Theoretically, the addition of the bridge's velocity feedback to control scheme (20) is capable of avoiding the self-excited vibration. However, it should be checked numerically and experimentally prior to entry into commercial service.

5.1. Numerical Validation. To obtain creditable conclusions, the engineering conditions should be simulated at great length. Firstly, the overall nonlinear maglev vehicle-bridge model with detail, including the vehicle body, the secondary, the bogies and the levitation modules, the saturation of control voltages, the misalignment distribution between the actuators and sensors, is adopted. Besides, considering the eddy gap sensor is polluted by the high-frequency magnetic field, the reasonable amount of noise is applied to the gap sensors. Besides, the direct component of the acceleration transducer is set as $0.2 \mathrm{~m} / \mathrm{s}^{2}$.

In this subsection, the parameters of controller are set as $k_{p}=1000, k_{d}=30, k_{a}=0.4$, and $k_{B}=30$. Modal damping $\xi_{B}$ of bridge is set as 0.01 . To simulate the limit cycle observed in maglev engineering practice, the hard nonlinear damping of bridge is assumed and set as $\xi_{B}^{\text {non }}(t)=\xi_{B}+200 \cdot\left|q_{B}(\tau)\right|$. The simulation results are shown in Figure 9.

When the time sequence is less than $1 \mathrm{~s}$, expected levitation gap $\delta_{\text {set }}$ is linearly varied, with the help of the integration term, and the gap error decays to zero quickly. In this section, modal frequency $\omega_{B}$ is set as 81.68 , which belongs to unstable interval (67.3 118.7). In this case, the dynamic responses are shown in Figure 9.

Expectedly, the self-excited vibration occurs. The amplitude of electromagnet's acceleration is up to $2 \mathrm{~m} / \mathrm{s}^{2}$, which transfers to the vehicle and degrades the ride comfort. The fluctuation of levitation gap is about $0.5 \mathrm{~mm}$, which impacts the stability of the levitation system.

According to Figure 9(d), the estimated velocity is unavailable when $t<2 \mathrm{~s}$, which is the transient response of the velocity estimator. To show the validity, the improved control scheme is activated at $t=4 \mathrm{~s}$. Afterwards, the amplitude of the vibration is attenuated quickly, and the selfexcited vibration decays to zero finally.

5.2. Experimental Validation. The experiments were conducted on the maintenance platform of the maglev test line, as shown in Figure 10. The control system under test included a PWM chopper, a levitation module, and a Power PC based digital control system which is capable of executing complex levitation control and vibration control algorithms.

All the experimental data was acquired through the Ethernet based levitation monitoring network and a laptop based monitoring terminal. The data sampling rate was 200 samples per second.

Figure 11 shows the result of the field test that was undertaken on the maintenance platform where the self-excited vibration occurred. When $t<4$ s, the self-excited vibration occurs. It can be observed that the levitation gap, current, and acceleration signals fluctuate violently. The vibration of electromagnet degrades the stability of the levitation control, decreasing the ride comfort.

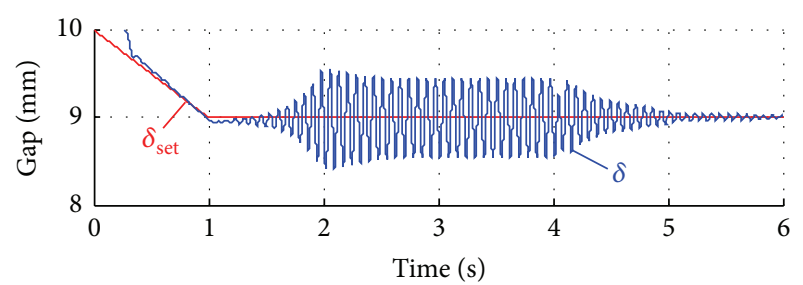

(a)

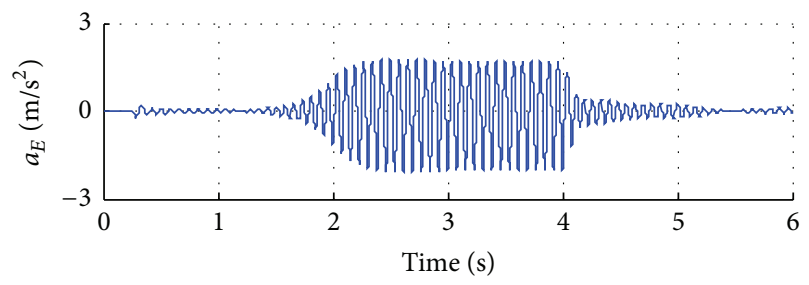

(b)

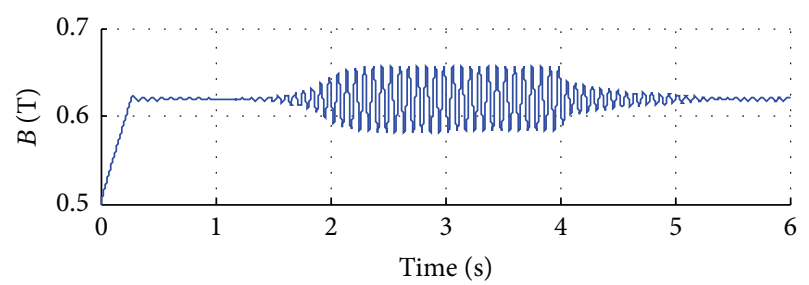

(c)

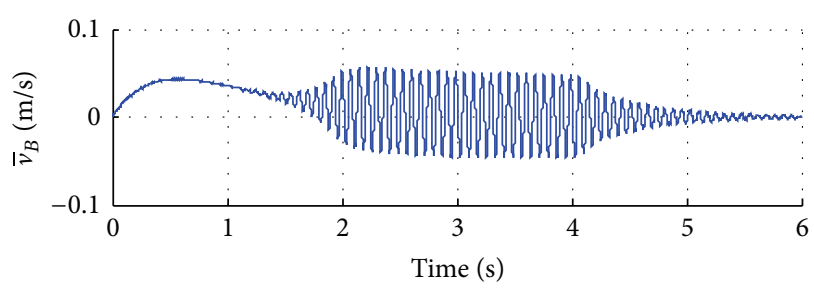

(d)

FIGURE 9: The numerical verification for vibration suppression method, which is activated at $t=4 \mathrm{~s}$; (a) the levitation gap and expected gap; (b) the acceleration of electromagnet; (c) the magnetic flux; (d) the estimated velocities of bridge.

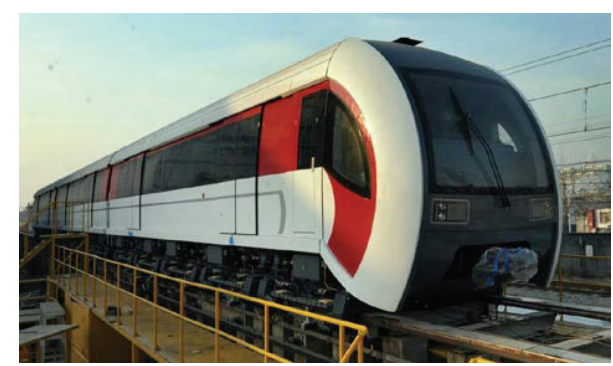

Figure 10: Field experiments on a full-scale maglev train at Tangshan maglev engineering base.

To check the validity, the improved control scheme was activated at $t=4 \mathrm{~s}$. After a more drastic and short regulation, the fluctuation of the levitation gap and the acceleration of electromagnet were attenuated greatly.

Considering that the maglev train consists of five bogies, we believe that the self-excited vibration will die away at last 


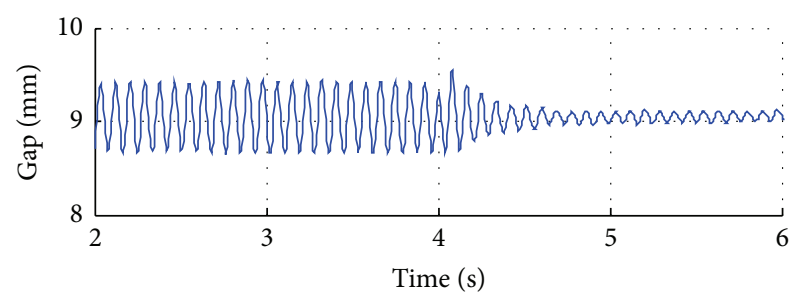

(a)

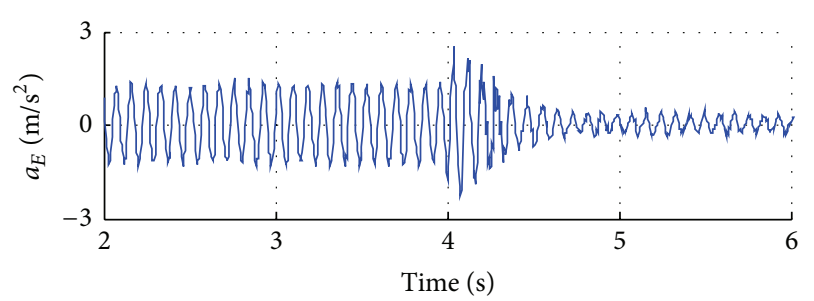

(b)

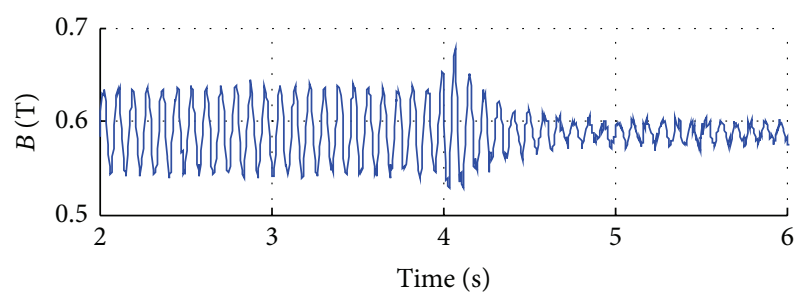

(c)

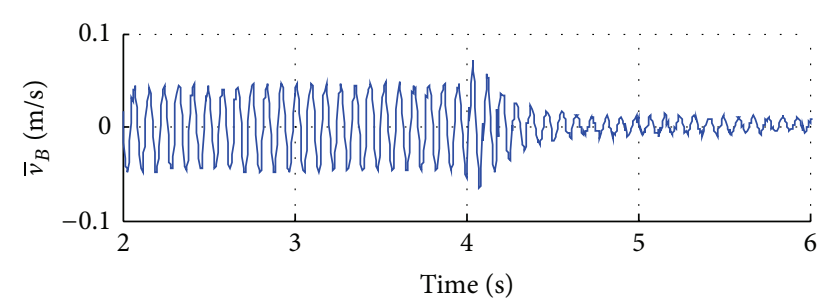

(d)

FIGURE 11: The experimental verification for vibration suppression method, which is activated at $t=4 \mathrm{~s}$; (a) the levitation gap and expected gap; (b) the acceleration of electromagnet; (c) the magnetic flux; (d) the estimated velocities of bridge.

if the proposed suppression method of vibration is applied to five bogies and all activated at $t=4 \mathrm{~s}$.

\section{Conclusions}

Firstly, a minimum model containing a flexible bridge and ten levitation units is presented. Based on the minimum model, we conclude that the magnetic flux feedback is capable of simplifying the block diagram of the vehicle-bridge interaction system. Secondly, considering the uncertainty of the bridge's modal frequency, the stability of the levitation system itself and the interaction system are explored according to the real parts of the three characteristic roots. Furthermore, motivated by the positive effects of the mechanical damping of bridges and the feedback channels' difference between the levitation subsystem and the bridge subsystem, the increment of electrical damping by the additional feedback of vertical velocity of bridge is proposed and several related implementation issues are addressed. Finally, the numerical and experimental validities illustrating the stability improvements are carried out.

\section{Competing Interests}

The authors declare that they have no competing interests.

\section{Acknowledgments}

This work was financially supported in part by the National Natural Science Foundation of China under Grant 112002230 and Grant 11302252.

\section{References}

[1] H.-W. Lee, K.-C. Kim, and J. Lee, "Review of Maglev train technologies," IEEE Transactions on Magnetics, vol. 42, no. 7, pp. 1917-1925, 2006.

[2] L. G. Yan, "Development and application of the Maglev transportation system," IEEE Transactions on Applied Superconductivity, vol. 18, no. 2, pp. 92-99, 2008.

[3] D. M. Rote and Y. Cai, "Review of dynamic stability of repulsive-force maglev suspension systems," IEEE Transactions on Magnetics, vol. 38, no. 2, pp. 1383-1390, 2002.

[4] H.-B. Zhou and J.-A. Duan, "A novel levitation control strategy for a class of redundant actuation maglev system," Control Engineering Practice, vol. 19, no. 12, pp. 1468-1478, 2011.

[5] R.-J. Wai and J.-D. Lee, "Robust levitation control for linear Maglev rail system using fuzzy neural network," IEEE Transactions on Control Systems Technology, vol. 17, no. 1, pp. 4-14, 2009.

[6] D. F. Zhou, C. H. Hansen, and J. Li, "Suppression of maglev vehiclegirder self-excited vibration using a virtual tuned mass damper," Journal of Sound and Vibration, vol. 330, no. 5, pp. 883901, 2011.

[7] T. E. Alberts and G. Oleszczuk, "On the influence of structural flexibility on feedback control system stability for EMS Maglev vehicles," in Proceedings of the 19th International Conference on Magnetically Levitated Systems and Linear Drives, Dresden, Germany, September 2006.

[8] T. E. Alberts and A. Hanasoge, "Modeling and experimental validation of an EMS demonstration vehicle," in Proceedings of the 20th International Conference on Magnetically Levitated Systems and Linear Drives, San Diego, Calif, USA, December 2008.

[9] H. P. Wang, J. Li, and K. Zhang, "Sup-resonant response of a nonautonomous maglev system with delayed acceleration feedback control," IEEE Transactions on Magnetics, vol. 44, no. 10, pp. 2338-2350, 2008.

[10] L. Zhang, Z. Zhang, and L. Huang, "Double Hopf bifurcation of time-delayed feedback control for maglev system," Nonlinear Dynamics, vol. 69, no. 3, pp. 961-967, 2012.

[11] L. Zhang, L. Huang, and Z. Zhang, "Hopf bifurcation of the maglev time-delay feedback system via pseudo-oscillator analysis," Mathematical and Computer Modelling, vol. 52, no. 56, pp. 667-673, 2010.

[12] J.-H. Li, J. Li, D.-F. Zhou, and P.-C. Yu, "Self-excited vibration problems of maglev vehicle-bridge interaction system," Journal of Central South University, vol. 21, no. 11, pp. 4184-4192, 2014. 
[13] S. T. Pang, T.-C. Tsao, and L. A. Bergman, "Active and passive damping of Euler-Bernoulli beams and their interactions," Journal of Dynamic Systems, Measurement and Control, vol. 115, no. 3, pp. 379-384, 1993.

[14] H. J. Hong and Y. G. Li, "The design of a magnetic levitation controller based on the study of coupling vibration," in Proceedings of the 18th International Conference on Magnetically Levitated Systems and Linear Drives, pp. 1012-1018, Shanghai, China, 2004.

[15] H. S. Han, B. Y. Yim, N. J. Lee, Y. C. Hur, and J. G. Kwon, "Vibration analysis of a maglev vehicle using electromagnetic suspension," in Proceedings of the International Conference on Electrical Machines and Systems (ICEMS '07), pp. 1963-1969, Seoul, Republic of Korea, October 2007.

[16] H. S. Han, B. H. Yim, N. J. Lee, Y. C. Hur, and S. S. Kim, "Effects of the guideway's vibrational characteristics on the dynamics of a Maglev vehicle," Vehicle System Dynamics, vol. 47, no. 3, pp. 309-324, 2009.

[17] J. H. Li, J. Li, D. F. Zhou, and L. C. Wang, "The modeling and analysis for the self-excited vibration of the maglev vehiclebridge interaction system," Mathematical Problems in Engineering, vol. 2015, Article ID 709583, 10 pages, 2015.

[18] J. H. Li, J. Li, D. F. Zhou, P. Cui, L. C. Wang, and P. C. Yu, "The active control of maglev stationary self-excited vibration with a virtual energy harvester," IEEE Transactions on Industrial Electronics, vol. 62, no. 5, pp. 2942-2951, 2015.

[19] E. Kong, J.-S. Song, B.-B. Kang, and S. Na, "Dynamic response and robust control of coupled maglev vehicle and guideway system," Journal of Sound and Vibration, vol. 330, no. 25, pp. 6237-6253, 2011.

[20] J.-S. Lee, S.-D. Kwon, M.-Y. Kim, and I. H. Yeo, "A parametric study on the dynamics of urban transit maglev vehicle running on flexible guideway bridges," Journal of Sound and Vibration, vol. 328, no. 3, pp. 301-317, 2009. 


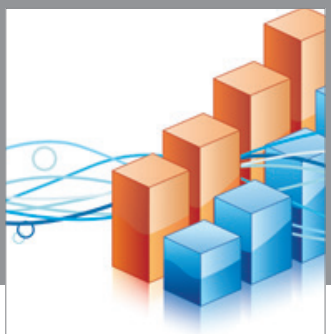

Advances in

Operations Research

vatem alat4

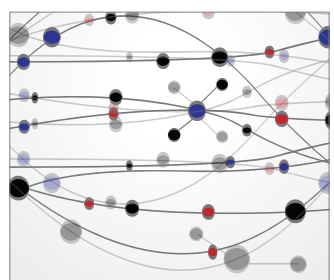

\section{The Scientific} World Journal
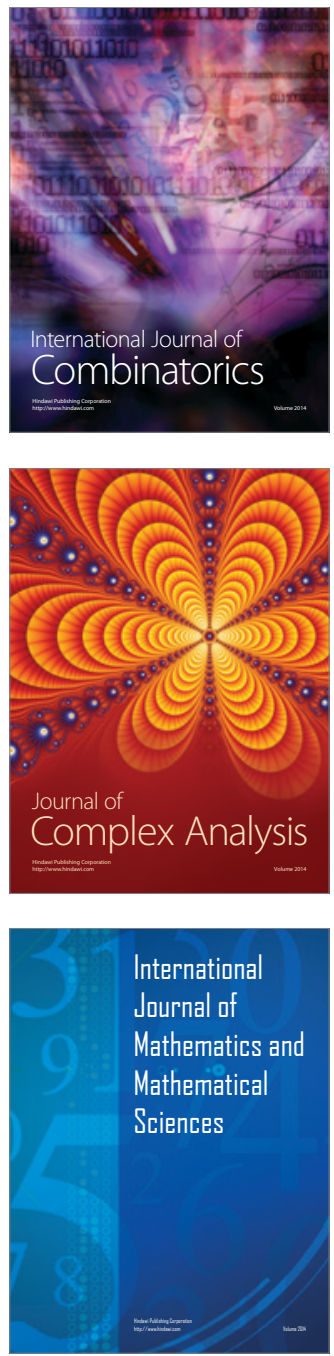
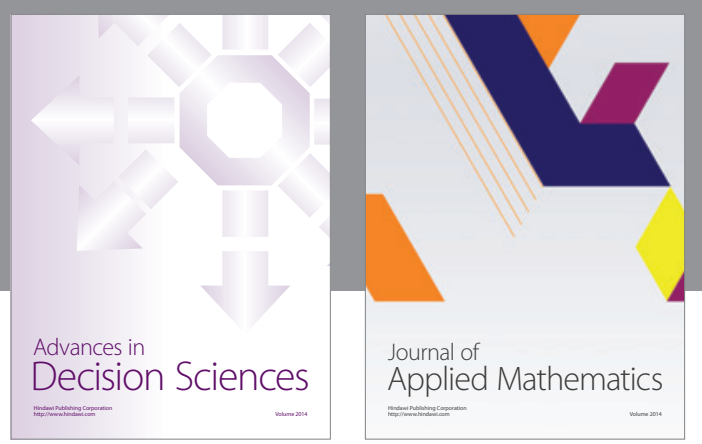

Algebra

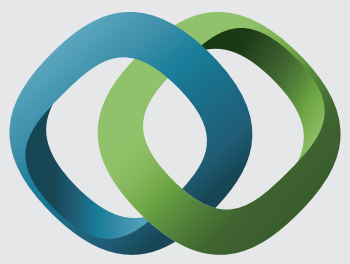

\section{Hindawi}

Submit your manuscripts at

http://www.hindawi.com
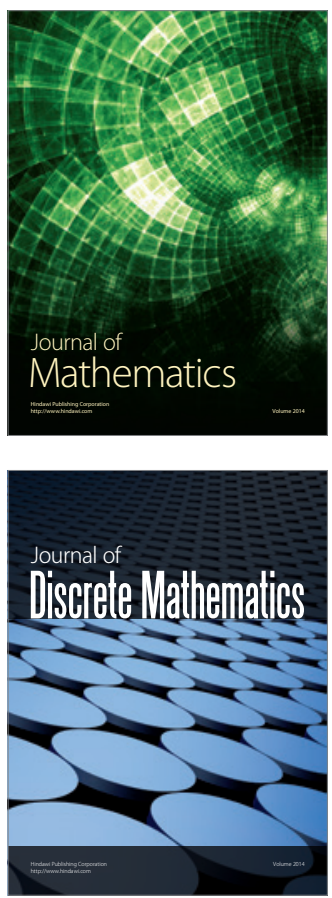

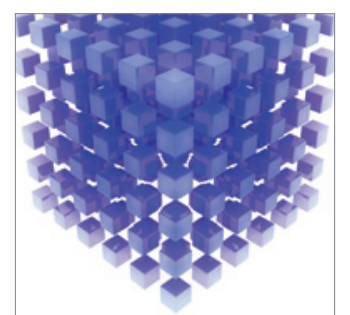

Mathematical Problems in Engineering
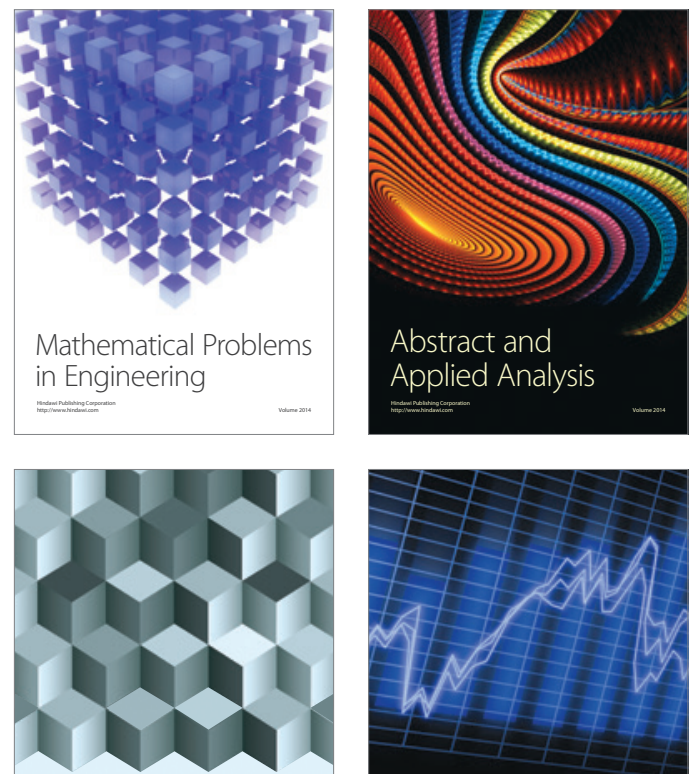

Journal of

Function Spaces

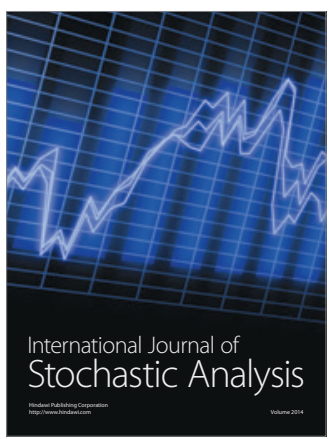

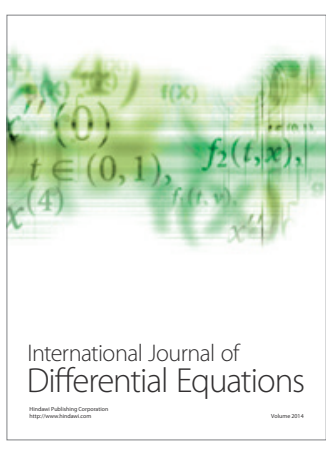
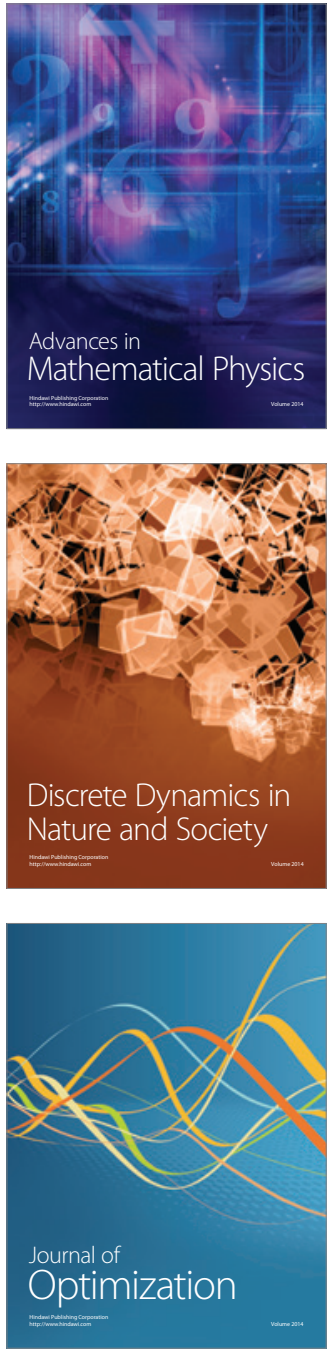